\title{
Diophantine Equations and Linear Recurrences
}

\section{MAHADI DDAMUliRA}

Institute of Analysis and Number Theory

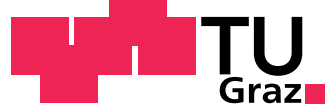

Graz University of Technology

Ph.D. Thesis Defense

Supervisors: Robert TICHY and Florian LUCA (Johannesburg)
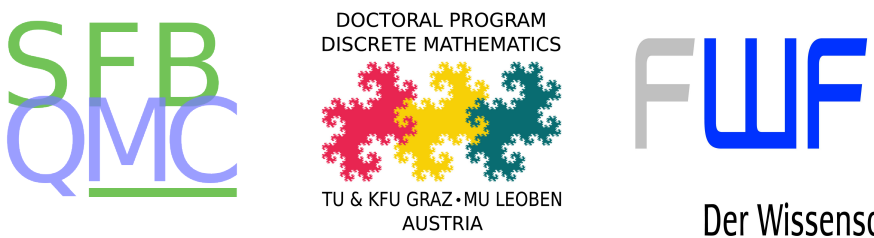

Der Wissenschaftsfonds.

Graz, 5 June 2020 


\section{Overview of Our Work}

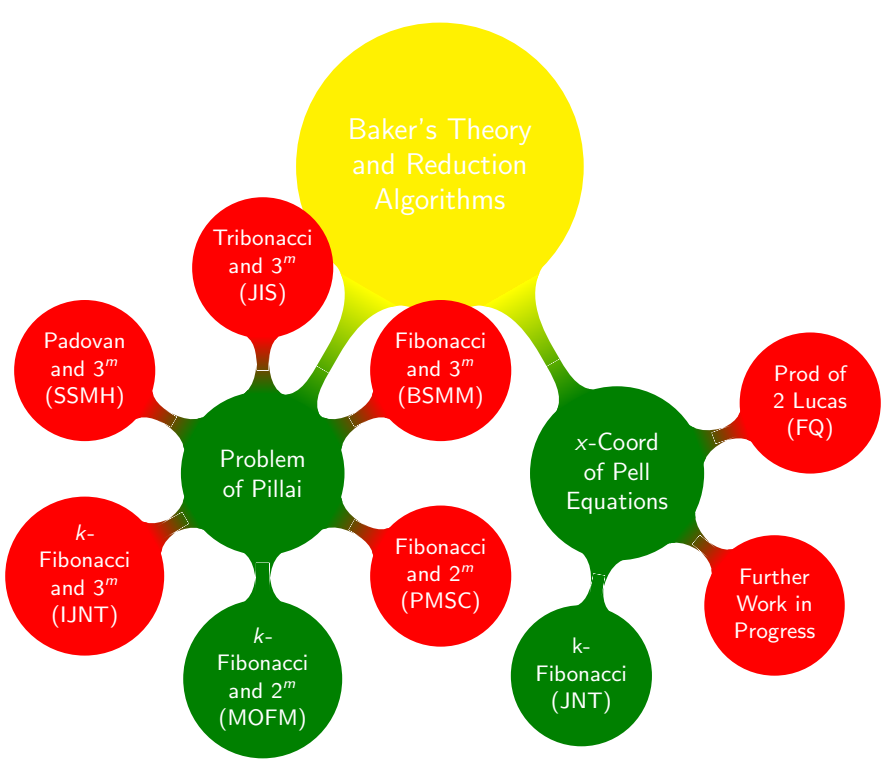




\section{Outline}

(1) Problem of Pillai

(2) $x$-Coordinates of Pell Equations

(3) Baker's Methods

(4) Reduction Procedure

(5) Illustration of the proofs 


\section{Problem of Pillai}


- Pillai's problem asks to prove that, for any positive integer $c$, there exist finitely many positive integers $a, b, x, y$ with $\min \{x, y\} \geq 2$ such that

$$
a^{x}-b^{y}=c .
$$

- It was conjectured by Pillai in 1936, and proved by Stroecker and Tijdeman in 1982, that the only integers $c$ admitting at least two representations of the form $2^{x}-3^{y}$ are

$$
\begin{aligned}
& 2^{3}-3^{2}=2^{1}-3^{1}=-1 ; \quad 2^{5}-3^{3}=2^{3}-3^{1}=5 \\
& 2^{8}-3^{5}=2^{4}-3^{1}=13
\end{aligned}
$$

- While many other cases have been studied - including the famous case $c=1$ (Catalan's problem), resolved by Mihăilescu in 2006 Pillai's problem remains open in general. 


\section{k-generalized Fibonacci numbers}

- For an integer $k \geq 2$, the $k$-generalized Fibonacci sequence is defined by the recurrence relation

$$
F_{n}^{(k)}=F_{n-1}^{(k)}+F_{n-2}^{(k)}+\cdots+F_{n-k}^{(k)},
$$

with the initial conditions

$$
F_{-(k-2)}^{(k)}=F_{-(k-3)}^{(k)}=\cdots=F_{0}^{(k)}=0, \quad F_{1}^{(k)}=1 .
$$

- The case $k=2$ recovers the classical Fibonacci sequence, the case $k=3$ recovers the Tribonacci sequence, etc.

- The generalized Fibonacci analogue of Pillai's problem is concerned with the study, for any fixed positive integers $k, \ell, c$, of the solutions in positive integers $n, m$, to the equation

$$
F_{n}^{(k)}-F_{m}^{(\ell)}=c .
$$




\section{Recent results I}

Particular cases have been studied: $(n, m) \neq\left(n_{1}, m_{1}\right)$.

- D.-Luca-Rakotomalala (2017)

$$
F_{n}-2^{m}=F_{n_{1}}-2^{m_{1}}=c ; \quad c \in\{-30,-11,-3,0,1,5,85\} .
$$

- Bravo-Luca-Yazán (2017)

$$
T_{n}-2^{m}=T_{n_{1}}-2^{m_{1}}=c ; \quad c \in\{-8,-3,-1,0,5\} .
$$

- Chim-Pink-Ziegler (2017)

$$
F_{n}-T_{m}=F_{n_{1}}-T_{m_{1}}=c ; \quad c \in
$$

$\{-271,-60,-41,-23,-22,-11,-10,-5,-3,-2,-1,0,1,4,6,8,11\}$. 


\section{Recent results II}

- D. (2019)

$$
F_{n}-3^{m}=F_{n_{1}}-3^{m_{1}}=c ; \quad c \in\{-26,-6,-1,0,2,4,7,12\} .
$$

- D. (2019)

$$
T_{n}-3^{m}=T_{n_{1}}-3^{m_{1}}=c ; \quad c \in\{-2,0,1,4\} .
$$

- D. (2019)

$$
P_{n}-3^{m}=P_{n_{1}}-3^{m_{1}}=c ; \quad c \in\{-6,0,1,22,87\} .
$$

$\left\{P_{n}\right\}_{n \geq 0}$ - Padovan sequence given by $P_{0}=0, P_{1}=1=P_{2}$, and $P_{n+3}=P_{n+1}+P_{n}$ for all $n \geq 0$.

- Similar problems have been solved by several other authors... 


\section{Main results I}

- D.-Gómez-Luca (2018)

Assume that $k \geq 4$. Then, the Diophantine equation $F_{n}^{(k)}-2^{m}=F_{n_{1}}^{(\bar{k})}-2^{m_{1}}=c$ with $n>n_{1} \geq 2, m>m_{1} \geq 0$ has the following families of solutions ( $\left.c, n, m, n_{1}, m_{1}\right)$.

(i) In the range $2 \leq n_{1}<n \leq k+1$, we have the following solution:

$$
(0, s, s-2, t, t-2) \quad \text { for } \quad 2 \leq t<s \leq k+1 .
$$

(ii) In the ranges $2 \leq n_{1} \leq k+1$ and $k+2 \leq n \leq 2 k+2$, we have the following solutions:

(a) when $n_{1}=n-1$ :

$$
\left(2^{k-1}-1, k+2, k-1, k+1,0\right)
$$




\section{Main results II}

(b) when $n_{1}<n-1$ :

$$
\left(2^{\gamma}-2^{\rho}, k+2^{a}-2^{b}, k+2^{a}-2^{b}-2, \gamma+2, \rho\right),
$$

with $\gamma=b-3+2^{a}-2^{b}$ and $\rho=a-3+2^{a}-2^{b}$, where $a>b \geq 0$, $(a, b) \neq(1,0)$ and $\gamma+3 \leq k+2$.

(iii) In the range $k+2 \leq n_{1}<n \leq 2 k+2$, we have the following solutions: if the integer $a$ is maximal such that $2^{a} \leq k+2$ satisfies $a+2^{a}=k+1+2^{b}$ for some positive integer $b$, then

$$
\left(-2^{a+2^{a}-3}, k+2^{a}, k+2^{a}-2, k+2^{b}, b+2^{b}-3\right) .
$$

(iv) If $n=2 k+3$, and additionally $k=2^{t}-3$ for some integer $t \geq 3$, then:

$$
\left(1-2^{t+2^{t}-3}, 2^{t+1}-3,2^{t+1}-5,2, t+2^{t}-3\right) .
$$

The equation has no solutions with $n>2 k+3$. 


\section{Main results III}

\section{Theorem (D.-Luca, 2020)}

Let $k \geq 4$ be fixed. Then, $F_{n}^{(k)}-3^{m}=F_{n_{1}}^{(k)}-3^{m_{1}}=c$, with $n>n_{1} \geq 2$ and $m>m_{1} \geq 1$ has solutions with

(i) $c \in\{-1,5,13\}$ and $2 \leq n \leq k+1$ as follows: (cf. Pillai 1936)

$$
\begin{aligned}
& F_{5}^{(k)}-3^{2}=F_{3}^{(k)}-3^{1}=-1, \quad k \geq 4, \\
& F_{7}^{(k)}-3^{3}=F_{5}^{(k)}-3^{1}=5, \quad k \geq 6, \\
& F_{10}^{(k)}-3^{5}=F_{6}^{(k)}-3^{1}=13, \quad k \geq 9
\end{aligned}
$$

(ii) $c \in\{-25,-7,5\}$ and $n \geq k+2$ and $k \in\{4,5,6\}$ as follows:

$$
\begin{aligned}
& F_{8}^{(4)}-3^{4}=F_{3}^{(4)}-3^{3}=-25 \\
& F_{10}^{(5)}-3^{5}=F_{3}^{(5)}-3^{2}=-7, \\
& F_{10}^{(6)}-3^{5}=F_{6}^{(6)}-3^{1}=5 .
\end{aligned}
$$




\section{$x$-Coordinates of Pell Equations}




\section{Pell Equations}

- Let $d \geq 2$ be a positive integer which is not a perfect square. It is well known that the Pell equation

$$
x^{2}-d y^{2}= \pm 1
$$

has infinitely many positive integer solutions $(x, y)$.

- By putting $\left(x_{1}, y_{1}\right)$ for the smallest such solution, all solutions are of the form $\left(x_{n}, y_{n}\right)$ for some positive integer $n$, where

$$
x_{n}+y_{n} \sqrt{d}=\left(x_{1}+y_{1} \sqrt{d}\right)^{n} \quad \text { for all } n \geq 1 .
$$

- Furthermore, the sequence $\left\{x_{n}\right\}_{n \geq 1}$ is binary recurrent. The following formula

$$
x_{n}=\frac{\left(x_{1}+y_{1} \sqrt{d}\right)^{n}+\left(x_{1}-y_{1} \sqrt{d}\right)^{n}}{2},
$$

holds for all positive integers $n$. 


\section{Some recent results}

- Luca-Togbé (2018)

$$
x_{n}=F_{m}
$$

Eq. (7) has at most one solution in positive integers except for $d=2$ with $(n, m)=(1,1),(1,2),(2,4)$.

- Luca-Montejano-Szalay-Togbé (2017)

$$
x_{n}=T_{m}
$$

Eq. (8) has at most one solution in positive integers except for: $d=2$ with $(n, m)=(1,1),(1,2),(3,5)$, and $d=3$ with $(n, m)=(1,3),(2,5)$. 


\section{Main results I}

Put $\epsilon:=x_{1}^{2}-d y_{1}^{2}$. Note that $d y_{1}^{2}=x_{1}^{2}-\epsilon$, so the pair $\left(x_{1}, \epsilon\right)$ determines $d$ and $y_{1}$.

\section{Theorem (D.-Luca, 2020)}

Let $k \geq 4$ be a fixed integer. Let $d \geq 2$ be a square-free integer. Assume that

$$
x_{n_{1}}=F_{m_{1}}^{(k)}, \quad \text { and } \quad x_{n_{2}}=F_{m_{2}}^{(k)}
$$

for positive integers $m_{2}>m_{1} \geq 2$ and $n_{2}>n_{1} \geq 1$, where $x_{n}$ is the $x$-coordinate of the nth solution of the Pell equation (4). Then, either:

(i) $n_{1}=1, n_{2}=2, m_{1}=(k+3) / 2, m_{2}=k+2$ and $\epsilon=1$; or

(ii) $n_{1}=1, n_{2}=3, k=3 \times 2^{a+1}+3 a-5, m_{1}=3 \times 2^{a}+a-1, m_{2}=$ $9 \times 2^{a}+3 a-5$ for some positive integer $a$ and $\epsilon=1$. 


\section{Main results II}

$\left\{L_{n}\right\}_{n \geq 0}$ - sequence of Lucas numbers given by $L_{0}=2, L_{1}=1$ and $L_{n+2}=L_{n+1}+L_{n}$ for all $n \geq 0$.

Consider the Diophantine equation

$$
x_{k}=L_{n} L_{m},
$$

in nonnegative integers $(k, n, m)$ with $k \geq 1$ and $0 \leq m \leq n$.

\section{Theorem (D., 2020)}

For each square-free integer $d \geq 2$, there is at most one integer $k$ such that the equation (10) holds, except for $d \in\{2,3,5,15,17,35\}$ for which $x_{1}=1, x_{2}=3, x_{3}=7, x_{9}=1393($ for $d=2), x_{1}=2, x_{2}=7($ for $d=3)$, $x_{1}=2, x_{2}=9($ for $d=5), x_{1}=4, x_{5}=15124$ (for $\left.d=15\right)$, $x_{1}=4, x_{2}=33($ for $d=17)$, and $x_{1}=6, x_{3}=846($ for $d=35)$. 


\section{Baker's Methods}




\section{Effective bounds I}

Let $\eta$ be an algebraic number of degree $d$ with minimal primitive polynomial over the integers

$$
a_{0} x^{d}+a_{1} x^{d-1}+\cdots+a_{d}=a_{0} \prod_{i=1}^{d}\left(x-\eta^{(i)}\right),
$$

where the leading coefficient $a_{0}$ is positive and the $\eta^{(i)}$ 's are the conjugates of $\eta$. Then the logarithmic height of $\eta$ is given by

$$
h(\eta):=\frac{1}{d}\left(\log a_{0}+\sum_{i=1}^{d} \log \left(\max \left\{\left|\eta^{(i)}\right|, 1\right\}\right)\right) .
$$

Useful properties of the logarithmic height function $h(\cdot)$ :

$$
\begin{aligned}
h\left(\eta_{1} \pm \eta_{2}\right) & \leq h\left(\eta_{1}\right)+h\left(\eta_{2}\right)+\log 2, \\
h\left(\eta_{1} \eta_{2}^{ \pm 1}\right) & \leq h\left(\eta_{1}\right)+h\left(\eta_{2}\right), \\
h\left(\eta^{s}\right) & =|s| h(\eta) \quad(s \in \mathbb{Z}) .
\end{aligned}
$$




\section{Effective bounds II}

\section{Theorem (Matveev according to Bugeaud, Mignotte, Siksek)}

Let $\eta_{1}, \ldots, \eta_{t}$ be positive real numbers in a number field $\mathbb{K}$ of degree $D$, let $b_{1}, \ldots, b_{t}$ be nonzero integers, and assume that $\Lambda:=\eta_{1}^{b_{1}} \cdots \eta_{t}^{b_{t}}-1$, is nonzero. Then

$$
\log |\Lambda|>-1.4 \times 30^{t+3} \times t^{4.5} \times D^{2}(1+\log D)(1+\log B) A_{1} \cdots A_{t},
$$

where

$$
B \geq \max \left\{\left|b_{1}\right|, \ldots,\left|b_{t}\right|\right\},
$$

and

$$
A_{i} \geq \max \left\{D h\left(\eta_{i}\right),\left|\log \eta_{i}\right|, 0.16\right\}, \quad \text { for all } \quad i=1, \ldots, t .
$$




\section{Effective bounds III}

For $t=2$ and $\eta_{1}, \eta_{2}$ are positive and multiplicatively independent. Let in this case $B_{1}, B_{2}$ be real numbers larger than 1 such that

$$
\log B_{i} \geq \max \left\{h\left(\eta_{i}\right), \frac{\left|\log \eta_{i}\right|}{D}, \frac{1}{D}\right\}, \quad \text { for } \quad i=1,2 .
$$

Put $b^{\prime}:=\frac{\left|b_{1}\right|}{D \log B_{2}}+\frac{\left|b_{2}\right|}{D \log B_{1}}$ and $\Gamma:=b_{1} \log \eta_{1}+b_{2} \log \eta_{2}$.

$\Gamma \neq 0$ because $\eta_{1}$ and $\eta_{2}$ are multiplicatively independent.

\section{Theorem (Laurent, Mignotte, Nesterenko)}

With the above notations, assuming that $\eta_{1}, \eta_{2}$ are positive and multiplicatively independent, then

$\log |\Gamma|>-24.34 D^{4}\left(\max \left\{\log b^{\prime}+0.14, \frac{21}{D}, \frac{1}{2}\right\}\right)^{2} \log B_{1} \log B_{2}$ 


\section{Reduction Procedure}




\section{Diophantine approximation}

Homogeneous linear form in two $\mathbb{Z}$-variables: Classical result in the theory of Diophantine approximation.

\section{Lemma (Legendre)}

Let $\tau$ be an irrational number, $\frac{p_{0}}{q_{0}}, \frac{p_{1}}{q_{1}}, \frac{p_{2}}{q_{2}}, \ldots$ be all the convergents of the continued fraction of $\tau$, and $M$ be a positive integer. Let $N$ be a nonnegative integer such that $q_{N}>M$. Then, putting $a(M):=\max \left\{a_{i}: i=0,1,2, \ldots, N\right\}$, the inequality

$$
\left|\tau-\frac{r}{s}\right|>\frac{1}{(a(M)+2) s^{2}},
$$

holds for all pairs $(r, s)$ of positive integers with $0<s<M$. 


\section{Baker-Davenport}

Nonhomogeneous linear form in two $\mathbb{Z}$-variables: Baker-Davenport!

\section{Lemma (Dujella, Pethő)}

Let $M$ be a positive integer, $\frac{p}{q}$ be a convergent of the continued fraction of the irrational number $\tau$ such that $q>6 M$, and $A, B, \mu$ be some real numbers with $A>0$ and $B>1$. Furthermore, let $\varepsilon=\|\mu q\|-M\|\tau q\|$. If $\varepsilon>0$, then there is no solution to the inequality

$$
0<|u \tau-v+\mu|<A B^{-w},
$$

in positive integers $u, v$, and $w$ with

$$
u \leq M \quad \text { and } \quad w \geq \frac{\log (A q / \varepsilon)}{\log B} .
$$




\section{LLL algorithm}

\section{Lemma (LLL Algorithm)}

Let $X_{1}, X_{2}, \ldots, X_{t}$ be positive integers such that $X:=\max \left\{X_{i}\right\}$ and $C>(t X)^{t}$ is a fixed sufficiently large constant. Let $\Omega$ be a lattice, we consider a reduced base $\left\{\mathbf{b}_{i}\right\}$ to $\Omega$ and its associated Gram-Schmidt orthogonalization base $\left\{\mathbf{b}_{i}^{*}\right\}$. We set

$c_{1}=\max _{1 \leq i \leq t} \frac{\left\|\mathbf{b}_{1}\right\|}{\left\|\mathbf{b}_{i}^{*}\right\|}, \theta=\frac{\left\|\mathbf{b}_{1}\right\|}{c_{1}}, \quad Q=\sum_{i=1}^{t-1} X_{i}^{2}, \& R=\frac{1}{2}\left(1+\sum_{i=1}^{t} X_{i}\right)$.

If the integers $x_{i}$ are such that $\left|x_{i}\right| \leq X_{i}$, for $1 \leq i \leq t$ and $\theta^{2} \geq Q+R^{2}$, then we have

$$
\left|\sum_{i=1}^{t} x_{i} \tau_{i}\right| \geq \frac{\sqrt{\theta^{2}-Q}-R}{C} .
$$




\section{Illustration of the proofs}




$$
F_{n}^{(k)}-3^{m}=F_{n_{1}}^{(k)}-3^{m_{1}}(=c) .
$$

- Assume $(n, m) \neq\left(n_{1}, m_{1}\right)$. If $m=m_{1}$, then $F_{n}^{(k)}=F_{n_{1}}^{(k)}$. Since $\min \left\{n, n_{1}\right\} \geq 2$, then $n=n_{1}$. So, $(n, m)=\left(n_{1}, m_{1}\right)$, a contradiction! Thus, assume $n>n_{1} \geq 2$, then $m>m_{1} \geq 1$.

- Assume $2 \leq n_{1}<n \leq k+1$, then $F_{n_{1}}^{(k)}=2^{n_{1}-2}$ and $F_{n}^{(k)}=2^{n-2}$. $\Rightarrow \quad 2^{n-2}-3^{m}=2^{n_{1}-2}-3^{m_{1}}=c$ (cf. Classical Pillai).

- Assume $n \geq k+2$. Claim: $F_{n}^{(k)}-3^{m}=F_{n_{1}}^{(k)}-3^{m_{1}}$ and $2^{n-2}-3^{m}=2^{n_{1}-2}-3^{m_{1}}$ can not simultaneously hold. Idea: $2^{n-2}-F_{n}^{(k)}=2^{n_{1}-2}-F_{n_{1}}^{(k)}$. Sequence $\left\{2^{n-2}-F_{n}^{(k)}\right\}_{n \geq 2}$ is 0 at $2 \leq n \leq k+1,1$ at $n=k+2$, and increasing for $n>k+2$.

- Using $\left|F_{n}^{(k)}-\frac{\alpha-1}{2+(k+1)(\alpha-2)} \alpha^{n-1}\right|<1 / 2$ and $\alpha^{n-2} \leq F_{n}^{(k)} \leq \alpha^{n-1}$, we successively apply Matveev's theorem on (11) to bound $m, n$ polynomially in $k$. That is, $m \leq n<4 \times 10^{42} k^{11}(\log k)^{7}$. 
- The cutoff $k$. Asuume $n<4 \times 10^{42} k^{11}(\log k)^{7}<k^{k / 2}$. Thus, $k>600$. Two cases. The case of small $k, 4 \leq k \leq 600$ and the case of large $k, k>600$.

- For $4 \leq k \leq 600$. Very large bounds on $n$. We do Baker-Davenport! This reduces the bounds to $m \leq n \leq 500$.

With the help of Mathematica, we intersect the sets

$$
\begin{aligned}
F_{n, k} & :=\left\{F_{n}^{(k)}-F_{n_{1}}^{(k)}\left(\bmod 10^{20}\right): n \in[3,600], n_{1} \in[2, n-1]\right\}, \\
D_{n, k} & :=\left\{3^{m}-3^{m_{1}}\left(\bmod 10^{20}\right): m \in[2,600], m_{1} \in[1, m-1]\right\} .
\end{aligned}
$$

We get only the solutions listed in Theorem 1 .

- For $k>600$. Using $F_{n}^{(k)}=2^{n-2}(1+\zeta),|\zeta|<5 / 2^{k / 2}$, we again apply Matveev, Legendre criterion, and Baker-Davenport! The bounds obtained on $k, n, m$ lead to the case of small $k$. 


\section{End}

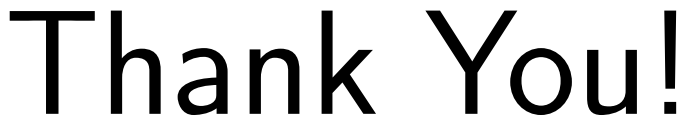

